\title{
Maximizando o Uso dos Recursos de GPU Através da Reordenação da Submissão de Kernels Concorrentes
}

\author{
Bernardo Breder ${ }^{1}$, Eduardo Charles ${ }^{1}$, Rommel Cruz ${ }^{1}$, Esteban Clua ${ }^{1}$, Cristiana Bentes ${ }^{2}$, \\ Lucia Drummond $^{1}$ \\ ${ }^{1}$ Instituto de Computação \\ Universidade Federal Fluminense (UFF), Rio de Janeiro, Brasil \\ ${ }^{2}$ Faculdade de Engenharia \\ Universidade do Estado do Rio de Janeiro (UERJ), Rio de Janeiro, Brasil \\ \{bbreder, eduardo, rquintanillac, esteban, lucia\}@ic.uff.br, \\ criseeng.uerj.br
}

\begin{abstract}
The increasing amount of resources available on current GPUs sparked new interest in the problem of sharing its resources by different kernels. While new generation of GPUs support concurrent kernel execution, their scheduling decisions are taken by the hardware at runtime. The hardware decisions, however, heavily depend on the order at which the kernels are submitted to execution. In this work, we propose a novel optimization approach to reorder the kernels invocation focusing on maximizing the resources utilization, improving the average turnaround time. We model the kernels assignments to the hardware resources as a series of knapsack problems and use dynamic programming approach to solve them. We evaluate our method using kernels with different sizes and resource requirements. Our results show significant gains in the average turnaround time and system throughput compared to the standard kernels submission implemented in modern GPUs.
\end{abstract}

Resumo. O aumento da quantidade de recursos disponíveis nas GPUs modernas despertou um novo interesse no problema do compartilhamento de seus recursos por diferentes kernels. A nova geração de GPUs permite a execução simultânea de kernels, porém ainda são limitadas ao fato de que decisões de escalonamento são tomadas pelo hardware em tempo de execução. Tais decisões dependem da ordem em que os kernels são submetidos para execução, criando execuções onde a GPU não necessariamente está com a melhor taxa de ocupação. Neste trabalho, apresentamos uma proposta de otimização para reordenar a submissão de kernels com foco em: maximizar a utilização dos recursos e melhorar o turnaround time médio. Modelamos a atribuição de kernels para a GPU como uma série de problemas da mochila e usamos uma abordagem de programação dinâmica para resolvê-los. Avaliamos nossa proposta utilizando kernels com diferentes tamanhos e requisitos de recursos. Nossos resultados mostram ganhos significativos no turnaround time médio e no throughput em comparação com a submissão padrão de kernels implementada em GPUs modernas. 


\section{Introdução}

Unidades de Processamento Gráfico (GPUs) estão cada vez mais populares nos dias de hoje devido ao seu maior desempenho e menor consumo de energia em comparação com as CPUs. GPUs suportam paralelismo maciço a custos relativamente baixos. No caso específico de GPUs da NVIDIA, no modelo de programação CUDA, kernels são disparados para a execução e executados por múltiplas threads. O programador organiza as threads em blocos e a GPU divide os núcleos em conjuntos de Streaming Multiprocessors (SMs). Os blocos de threads são criados em tempo de execução e o hardware atribui cada bloco a um SM específico.

Em face do aumento da quantidade de recursos disponíveis em GPUs e os avanços na sua microarquitetura, um novo tipo de problema começa a surgir: como compartilhar de forma eficaz os recursos da GPU por diferentes kernels? Muitas aplicações não estão prontas para tirar proveito de todos os recursos. De acordo com Pai et al. [Pai et al. 2013], o benchmark Parboil2 usa somente de $20 \%$ a $70 \%$ dos recursos da GPU Fermi. Adriaens et al. [Adriaens et al. 2012] realizaram estudos semelhantes para 12 aplicações reais e mostraram que a maioria delas exibem utilização desequilibrada de recursos GPU.

A fim de melhorar a utilização dos recursos, as nova gerações de GPUs da NVIDIA permitem a execução concorrente de kernels. Inicialmente introduzido na arquitetura Fermi, e posteriormente aprimorada na arquitetura Kepler, a execução concorrente de kernels permite que blocos de threads de diferentes kernels possam compartilhar os mesmos recursos da GPU.

A política de escalonamento de blocos de threads da GPU é implementada diretamente pelo hardware. Porém, a ordem com que os kernels são inseridos nas filas de escalonamento tem grande impacto na ocupação da GPU. Suponha o exemplo da Figura 1, onde quatro kernels são submetidos para executar em uma GPU. Cada kernel tem um tempo de execução e solicita uma quantidade diferente de recursos. Na Figura 1 (a), mostramos a execução dos kernels considerando a seguinte ordem de submissão: $\left\{k_{1}, k_{2}, k_{3}, k_{4}\right\}$. Podemos observar que de $t_{0}$ a $t_{1}$ há $40 \%$ dos recursos disponíveis e que em $t_{2}$ a $t_{3}$ há $50 \%$. Entretanto, se a ordem de submissão fosse $\left\{k_{4}, k_{1}, k_{3}, k_{2}\right\}$, obteríamos, conforme ilustrado na Figura 1 (b), um grande aumento no uso dos recursos.

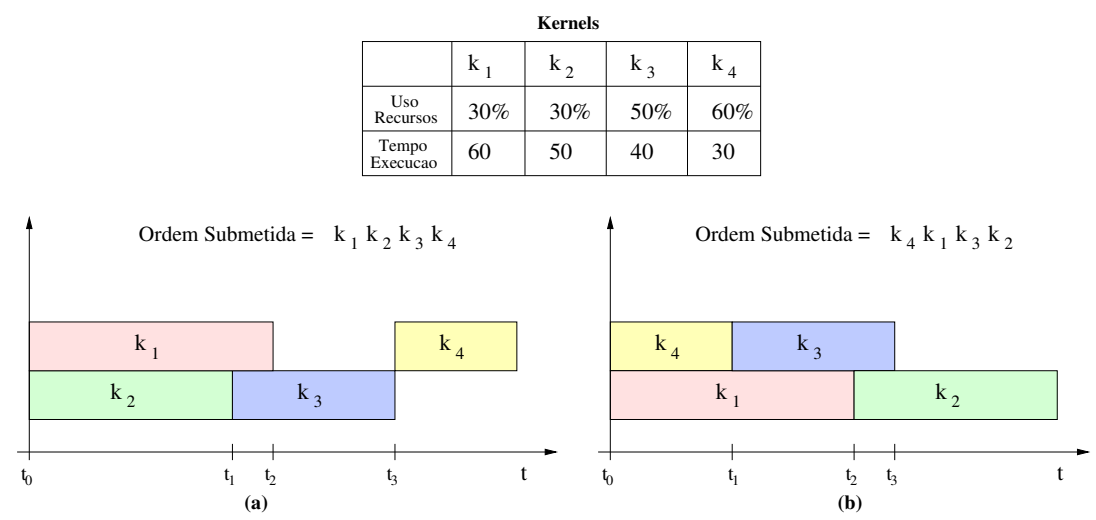

Figura 1. Um conjunto de 4 kernels submetidos à GPU em duas ordens diferentes. $\operatorname{Em~(a),~a~ordem~é~}\left\{k_{1}, k_{2}, k_{3}, k_{4}\right\}$. Em (b), a ordem é $\left\{k_{4}, k_{1}, k_{3}, k_{2}\right\}$

Neste trabalho, propomos uma nova estratégia de otimização que determina a me- 
lhor ordem para submeter kernels para a GPU. A ideia é simular a atribuição de kernels para as filas de hardware de modo a maximizar a utilização dos recursos, melhorando o throughput. Nossa proposta modela o problema de selecionar quais kernels são melhores para submissão para tirar o máximo proveito dos recursos disponíveis como uma série de problemas da mochila [Martello and Toth 1990]. Modelamos o conjunto dos recursos disponíveis como a capacidade da mochila e o conjunto de núcleos como os itens a serem inseridos na mochila. Os kernels têm pesos e valores de acordo com a quantidade de uso de recursos - memória compartilhada, número de registradores e número de threads - e o tempo de execução estimado. A solução para o problema da mochila estabelece o subconjunto de kernels cujo peso total é menor que os recursos disponíveis e o valor total é tão grande quanto possível. Usamos um algoritmo de programação dinâmica para resolver o problema da mochila e gerar uma nova ordem de submissão dos kernels.

Nosso algoritmo de reordenação é focado para uma tendência futura em que GPUs serão dispositivos multiprogramados, com um grande número de kernels compartilhando seus recursos. Apresentamos resultados de uma série de experimentos usando diferentes conjuntos de kernels com requisitos de recursos aleatórios. A nossa abordagem de reordenação foi capaz de reduzir o tempo médio de execução de $45 \%$ para $75 \%$ em comparação com a submissão de kernels padrão implementada em GPUs modernas. Também mostramos que o overhead referente ao cálculo de uma série de problemas da mochila é insignificante em comparação com os ganhos obtidos.

O restante do artigo é organizado da seguinte forma. A Seção 2 apresenta os trabalhos anteriores. A Seção 3 apresenta uma breve introdução do problema que estamos tratando. A Seção 4 descreve o algoritmo de reordenação proposto. A Seção 5 apresenta os resultados experimentais. Finalmente, na Seção 6, apresentamos nossas conclusões e propostas de trabalhos futuros.

\section{Trabalhos Relacionados}

Antes de as GPUs possuírem suporte de hardware para a execução simultânea de ker$n e l s$, alguns trabalhos propuseram superar esta limitação através da estratégia de software merging de diferentes kernels em um só [Peters et al. 2010, Wang et al. 2011, Gregg et al. 2012]. Em uma direção diferente, alguns autores propuseram modificar a granularidade dos kernels de modo a aumentar a utilização da GPU [Zhong and He 2014, Ravi et al. 2011, Pai et al. 2013]. Esta estratégia foi chamada de slicing por prover o fatiamento dos kernels de forma a se obter mais oportunidades de compartilhamento. Houve também alguns esforços em propor a divisão dos recursos da GPU entre os kernels concorrentes [Liang et al. 2015, Adriaens et al. 2012]. Estes esforços, no entanto, focaram em apontar melhorias no hardware da GPU para aumentar a sua utilização.

Mais próximo ao nosso trabalho estão as propostas que se concentram em obter melhor utilização com base na ordem em que os kernels são invocados no lado do host, chamado de reordenamento de kernels. Wende et al. [Wende et al. 2012] propuseram a reordenação para a arquitetura Fermi, onde a execução simultânea de kernels usa apenas uma fila de escalonamento. Eles criaram uma thread de escalonamento que organiza a fila de modo round-robin, sem considerar a utilização de recursos das threads. Os autores foram bem sucedidos no aumento da concorrência intercalando diferentes streams de execução na mesma fila, mas o hardware das GPUs modernas possui o 
mecanismo Hyper-Q, que implementa essas filas em hardware, possibilitando a execução realmente concorrente de kernels. O nosso trabalho é mais próximo ao trabalho de Li et $a l$. [Li et al. 2015] que propõe um esquema de reordenação para arquiteturas de GPU com tecnologia Hyper-Q. Sua abordagem calcula um score simbiótico, que tenta co-executar kernels com a utilização de recursos complementares e prevê o consumo de energia utilizando o conceito de rodadas de execução - rodada de execução simultânea de blocos de threads nos SMs. Eles tentam cumprir uma rodada com kernels simbióticos usando um algoritmo guloso para o problema bin-packing multidimensional. O uso de rodadas de execução simplifica as decisões de escalonamento. Em contraste, a nossa solução de reordenação evita a sincronização implícita na conclusão da rodada, resolvendo um problema mais complexo cada vez que há recursos disponíveis.

\section{Definição do Problema}

Para uma determinada GPU, $D$, seja $O r d=\left\{k_{0}, k_{1}, \ldots, k_{N-1}\right\}$ a lista de $N$ kernels independentes na ordem em que são submetidos para execução em $D$ ( $k_{i}$ é submetido antes de $k_{i+1}$ ). Dado que nossa estratégia de reordenação é executada de forma dinâmica, nossa solução pode ser usada em GPUs que estão constantemente recebendo novas demandas de execução. Neste sentido, Ord pode ser atualizada dinamicamente. Considere que $N S M$ é o número de SMs de $D$, e considere que D possui os recursos $R_{j} \mid j \in\{0,1,2\}$ :

- $R_{0}=$ tamanho da memória compartilhada por SM;

- $R_{1}=$ número de registradores por $\mathrm{SM}$;

- $R_{2}=$ número máximo de threads por SM.

A capacidade total de cada recurso de $D$ é definida por $W_{j}=R_{j} \times N S M \mid j \in$ $\{0,1,2\}$. Em um determinado instante de tempo durante a execução, há $m$ kernels executando concorrentemente em $D$, usando uma porcentagem de $W_{j}$. Os recursos disponíveis neste instante de tempo são chamados de $W_{j}^{a v} \mid j \in\{0,1,2\}$. Toda vez que um kernel termina e libera seus recursos, novos kernels são selecionados para execução de acordo com a quantidade de recursos disponíveis.

A fim de encontrar uma nova ordem de submissão, Ord ${ }^{\text {new }}$, maximizando a utilização de recursos, modelamos como um problema da mochila 0-1 a seleção de novos kernels para submissão. Neste modelo, no início da execução e a cada vez que um kernel terminar, $D$ representa a mochila com capacidade igual aos recursos disponíveis e os kernels representam os itens a serem empacotados na mochila. Para cada kernel $k_{i}$, as seguintes informações são coletadas:

- $t_{i}^{\text {est }}$ : tempo de execução estimado;

- $s h_{i}$ : quantidade de memória compartilhada requisitada por bloco;

- $n r_{i}$ : número de registradores requisitado por bloco;

- $n t_{i}$ : número máximo de threads disparadas por bloco;

- $n b_{i}$ : número de blocos.

Com relação ao tempo de execução estimado, há uma série de abordagens para realizar esta previsão com base em diferentes técnicas, tais como baseadas em simulação, machine learning e modelos analíticos [Lopez-Novoa et al. 2015]. Outra solução consiste na utilização de medidas de tempo de uma execução anterior da aplicação [Choi et al. 2013]. O estudo e a implementação de tais modelos de desempenho estão fora do escopo deste trabalho. 
As quantidades de memória compartilhada, registradores e threads podem ser obtidas de forma dinâmica através de diversas ferramentas, tais como o NVIDIA CUDA Profiler [NVIDIA 2016]. Atribuímos para cada kernel $k_{i}$ um peso $w_{i j}(i \in\{0, \ldots, N-1\}, j \in$ $\{0,1,2\}$, que representa a quantidade de recursos requisitados: $w_{i 0}=s h_{i} \times n b_{i}$, $w_{i 1}=n r_{i} \times n b_{i}$, e $w_{i 2}=n t_{i} \times n b_{i}$. Observe que, se $\exists j \mid w_{i j}>R_{j}, k_{i}$ ainda pode executar uma vez que a GPU pode escalonar um número de blocos de $k_{i}$ que cabem na GPU e, depois que terminar, escalonar o restante dos blocos. Neste caso, para considerar $k_{i}$ um item candidato, deve-se ajustar $w_{i j}=R_{j}$.

Atribuímos também um valor $v_{i}$ para cada kernel $k_{i}$ que representa a média do percentual dos recursos necessários por unidade de tempo. Kernels com tempos de execução menores terão maiores valores de $v_{i}$.

$$
v_{i}=\frac{\frac{s h_{i}}{R_{0}}+\frac{n r_{i}}{R_{1}}+\frac{n t_{i}}{R_{2}}}{3 \times t_{i}^{\text {est }}}
$$

Cada problema da mochila consiste em determinar a quantidade de kernels cuja soma do lucro é maximizada sem que a soma dos pesos exceda $W$. O problema da mochila é formulado como a maximização [Martello and Toth 1990]:

$$
\begin{gathered}
\text { maximize } \sum_{i=0}^{N-1} v_{i} x_{i} \\
\text { sujeito } a \sum_{i=0}^{N-1} w_{i j} x_{i} \leq W_{j} \mid j \in\{0,1,2\}
\end{gathered}
$$

onde $x_{i} \in\{0,1\}, x_{i}=1$ se o item $i$ deve ser incluído na mochila, e $x_{i}=0$ caso contrário. Neste problema, a maximização do lucro representa a maximização do uso de recursos.

\section{Proposta de Reordenação}

Basicamente, nossa proposta de reordenação simula a execução simultânea dos kernels. Definimos um número de filas $S$, tal que $S$ seja igual ao número de filas de escalonamento do hardware. Até $S$ kernels podem ser executados simultaneamente na GPU. O algoritmo considera que os kernels são escalonados pelo hardware de maneira round-robin enquanto existirem recursos disponíveis.

O Algoritmo 1 descreve a nossa abordagem. De forma geral, ele simula iterativamente o término do próximo kernel (que termina mais cedo), e encontra os próximos kernels para submeter através da resolução de um problema da mochila com os recursos disponíveis liberados pelo kernel que terminou.

Inicialmente, KernelList recebe todos os kernels em Ord $=\left\{k_{0}, k_{1}, \ldots, k_{N-1}\right\}$, e $W_{j}^{a v}=W_{j}, \forall j \in\{0,1,2\}$. A ordem Ord representa a ordem em que os kernels são invocados na aplicação. Enquanto houver kernels em KernelList, o algoritmo funciona da seguinte forma. A função GetKernelsToSubmit resolve um problema da mochila 0-1 e provê em NextSubmitList a lista de kernels em ordem descrescente de valores. Para cada kernel $k_{i}$ em NextSubmitList, o algoritmo insere $k_{i}$ na fila cujo tempo de término 
(término do último elemento) é o menor. Em seguida, os recursos necessários para $k_{i}$ são removidos de $W^{a v}$, e $k_{i}$ é removido de KernelList. O próximo passo é fazer avançar a execução e procurar o próximo término do kernel pela função SelectEarliestKernel. Esta função procura o kernel com o menor $t^{e s t}$. A conclusão do kernel é simulada através da inserção de seus recursos de volta para $W^{a v}$. No fim, o algoritmo cria $O r d^{\text {new }}$ através de uma busca round-robin nas filas.

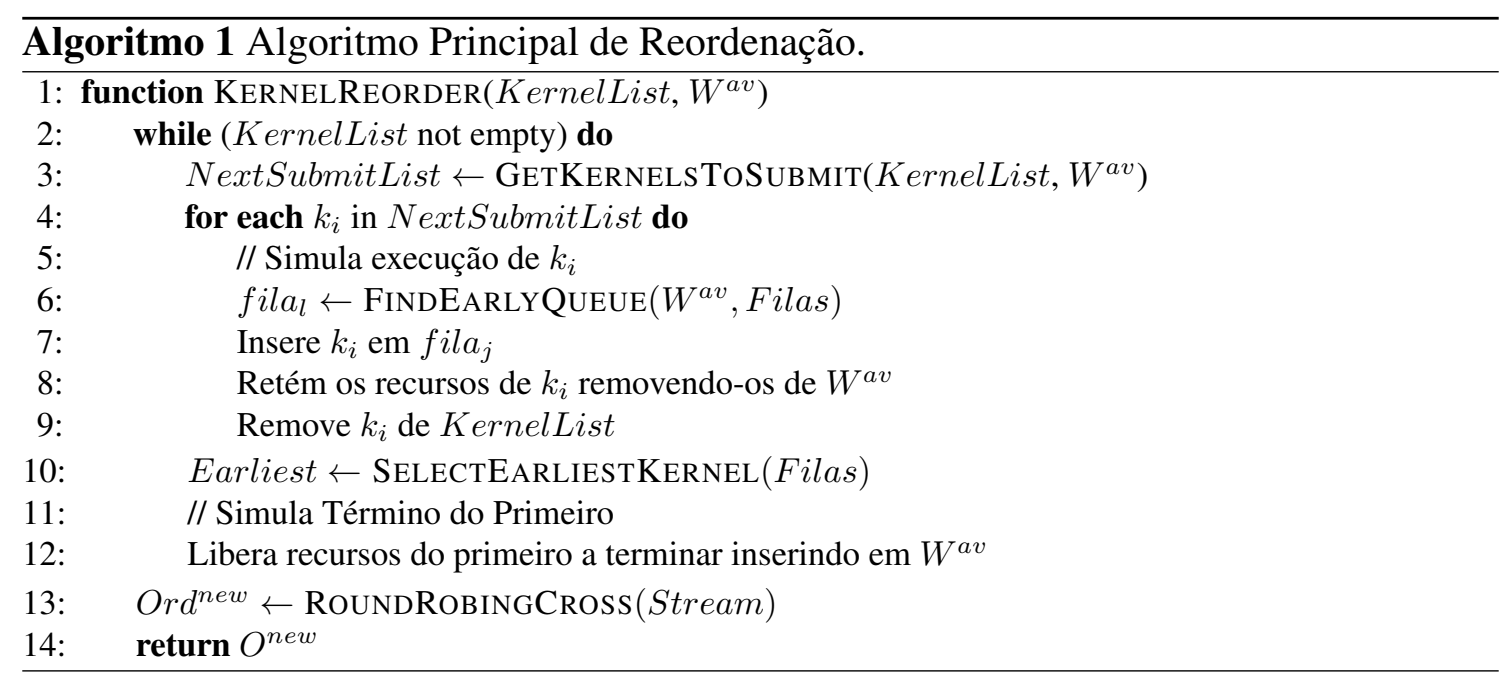

\subsection{Resolvendo o Problema da Mochila para Obter o Conjunto de Kernels}

Nossa solução para o problema da mochila multidimensional envolve a linearização dos pesos atribuídos aos kernels, de modo que eles sejam representados por um único inteiro. Calculamos um único valor $W$ que representa as três grandezas $W_{0}, W_{1}$ e $W_{2}$. Analogamente, os recursos necessários para cada kernel também são reduzidos a um único inteiro. Assim, $W=W_{0} \times W_{1} \times W_{2}$, e para cada kernel $k_{i}, w_{i}=\left(w_{i 0} \times W_{1} \times W_{2}\right)+\left(w_{i 1} \times\right.$ $\left.W_{2}\right)+w_{i 2}$.

O Algoritmo 2 apresenta o procedimento principal do método dinâmico utilizado para resolver o problema. Nas linhas 2 e 3, as variáveis SelectedKernels e tempW são inicializadas respectivamente com um conjunto vazio de kernels e com a capacidade total da GPU. Na linha 4, a função KnapsackMatrix é chamada com os parâmetros KernelList e $W^{a v}$. Esta função retorna uma matriz $M:(N+1) \times(W+1)$, onde as linhas representam os kernels e as colunas representam uma quantidade linearizada de recursos. Na linha 5, para cada kernel $k_{i}$, se houver recursos restantes, avalia-se a possibilidade de incluir $k_{i}$ através de consulta à matriz $M$. Na linha 7, o algoritmo testa se $k_{i}$ foi selecionado pelo método dinâmico. Se o valor da linha $i$ é diferente do valor prévio na coluna tempW (o que significa que a programação dinâmica selecionou $k_{i}$ ), ele é subtraído de $w_{i}$ e $k_{i}$ é incluído na lista SelectedKernels (linhas 8 e 9). No final, a lista NextSubmitList recebe os elementos de SelectedKernels ordenados em ordem descrescente de valor (linha 10).

A função KnapsackMatrix, que retorna a matriz $M$, é detalhada no Algoritmo 3. A matriz $M$ é inicializada na linha 3 e pode ser atualizada nas linhas 7 ou 9 . Na linha 7 , uma célula de $M$ é copiada para outra, o que significa que o kernel $k_{i}$ não é selecionado para ser incluído na mochila, porque excede a capacidade restante da mochila. Caso contrário, se ele gerar algum lucro, ele pode ser incluído na mochila (linha 9). 

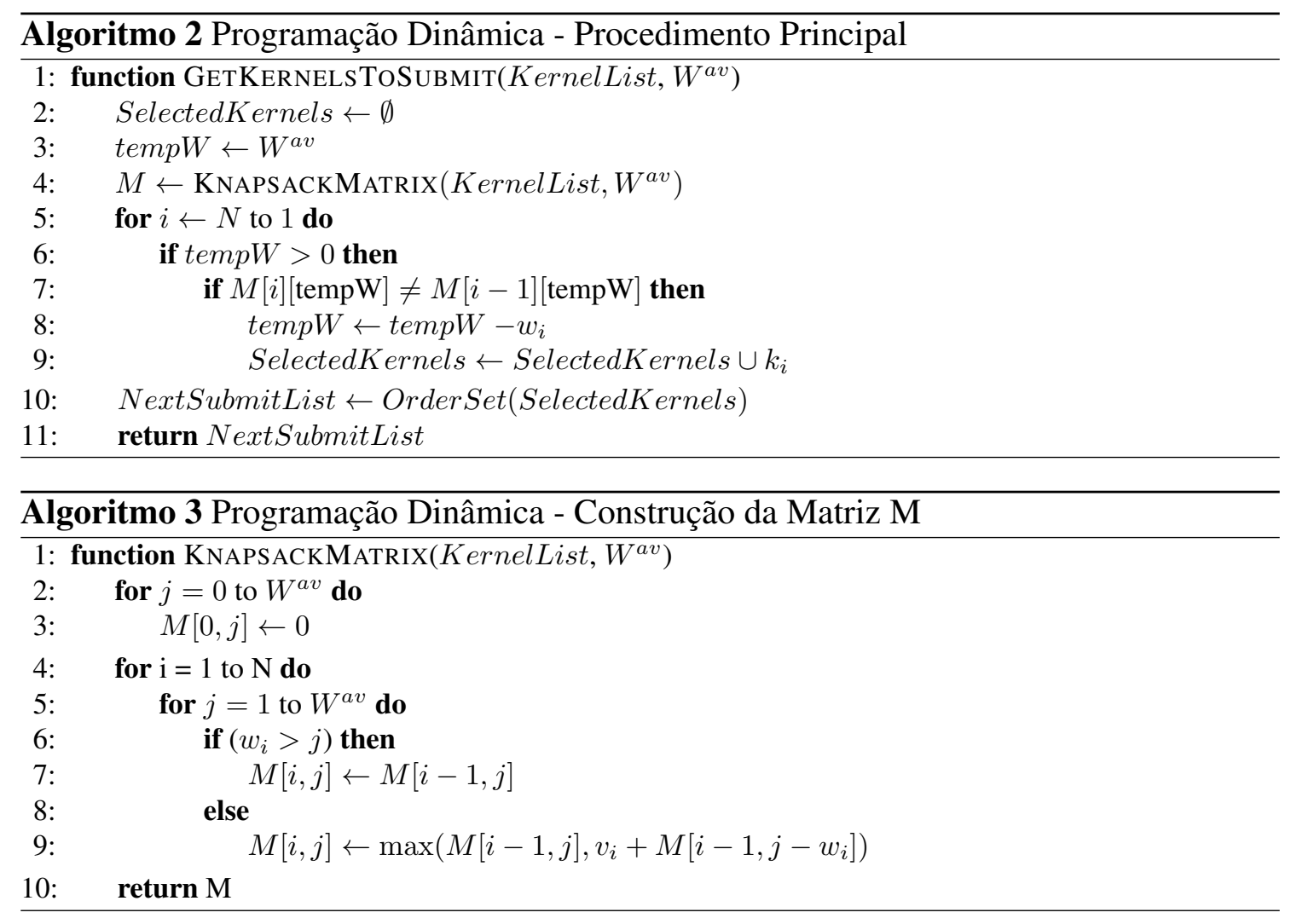

\section{Resultados Experimentais}

\subsection{Ambiente Computacional}

Utilizamos duas GPUs diferentes em nossos experimentos: TITAN X (arquitetura Maxwell) e Tesla K40 (arquitetura Kepler). Suas principais características estão apresentadas na Tabela 1. Os kernels foram implementados utilizando CUDA 7.5.

\begin{tabular}{|l|c|c|}
\hline & TITAN X & K40 \\
\hline Número de cores & 3.072 & 2.880 \\
\hline Clock do Core & $1000 \mathrm{MHz}$ & $745 \mathrm{MHz}$ \\
\hline RAM & $24 \mathrm{~GB}$ & $12 \mathrm{~GB}$ \\
\hline Bandapassante de Memória & $336,5 \mathrm{~GB} / \mathrm{s}$ & $288 \mathrm{~GB} / \mathrm{s}$ \\
\hline Capability & 5.2 & 3.5 \\
\hline Número de SMs & 24 & 15 \\
\hline Memória Compartilhada por SM & $96 \mathrm{~KB}$ & $48 \mathrm{~KB}$ \\
\hline Número de Registradores por SM & $64 \mathrm{~K}$ & $64 \mathrm{~K}$ \\
\hline Máx Num de Threads por SM & 2048 & 2048 \\
\hline Arquitetura & Maxwell & Kepler \\
\hline Performance no Pico & 6,6 TFLOPs & 4,2 TFLOPS \\
\hline
\end{tabular}

Tabela 1. Configurações das GPUs utilizadas.

\subsection{Metodologia}

Como a execução concorrente de kernels é uma característica relativamente nova em GPUs da NVIDIA, os programas em CUDA tendem a explorar o paralelismo de threads 
mais do que o paralelismo entre kernels. Acreditamos que no futuro, as GPUs vão funcionar como dispositivos multiprogramados, mas até o momento as aplicações de suites de benchmarks, como Rodinia, Parboil ou CUDA SDK, possuem um número muito pequeno de kernels independentes. Encontrar uma boa configuração de kernels com requerimentos de recursos realmente constrastantes é muito complicado nestes benchmarks. Portanto, decidimos avaliar nossa proposta de reordenação utilizando benchmarks sintéticos, em que podemos variar bastante e de forma controlada o número de kernels concorrentes e o requerimento de recursos.

Implementamos um gerador de kernel que cria conjuntos de $N$ kernels independentes em 32 CUDA streams para um dispositivo $D$ que possui os recursos $R_{0}, R_{1}$ e $R_{2}$. Os valores de $N$ e $R_{j} \mid j \in\{0,1,2\}$ são passados como parâmetros.

A estrutura geral de cada kernel sintético $k_{i}$ é de um código CUDA que executa um conjunto de operações aritméticas em uma matriz de números inteiros alocados na memória compartilhada. O número de blocos de $k_{i}$ e suas necessidades de recursos, $s h_{i}$, $n t_{i}$ e $t_{i}^{e s t}$, são criados randomicamente. Nos nossos experimentos, não estressamos o valor de $n r_{i}$, mas o mantivemos igual a zero.

Criamos diferentes experimentos com $N$ variando em $\{32,64,128,256\}$. Para cada valor de $N$, variamos também o número máximo e mínimo de blocos por kernel. O número mínimo de blocos é atribuído como 4 e o número máximo é aleatório mas limitado pelo valor de $N B$ que varia em $\{32,64,128,256,512\}$.

Para cada experimento com $N$ kernels, realizamos 50 execuções diferentes de modo a reduzir o impacto da aleatoriedade nos resultados. O gerador de kernels e o algoritmo de reordenação foram implementados em Java 1.7. Comparamos a nossa abordagem de reordenação com um cenário em que os kernels são submetidos à execução na mesma ordem em que foram invocadas, que chamamos de abordagem Padrão.

As métricas de avaliação utilizadas são: Average Normalized Turnaround Time (ANTT) e System Throughput (STP) propostas por Eyerman and Eeckhout em [Eyerman and Eeckhout 2008]. ANTT é uma métrica quanto menor melhor e mede o turnaround time médio normalizado pela execução do kernel sem concorrência. STP é uma métrica quanto maior melhor e mede a quantidade de tarefas completadas por unidade de tempo. Dado que o turnaround time de um kernel $k_{i}$ é representado por $T T_{i}$, e o turnaround time de $k_{i}$ quando executado sozinho é $T T_{i}^{S P}$ :

$$
A N T T=\frac{1}{N} \sum_{i=0}^{N-1} \frac{T T_{i}}{T T_{i}^{S P}} \quad \text { (4) } \quad S T P=\sum_{i=0}^{N-1} \frac{T T_{i}^{S P}}{T T_{i}}
$$

\subsection{Análise dos Resultados}

As Figuras 4 a 7 mostram os resultados de ANTT para diferentes valores de $N$ e diferentes valores de $N B$. Podemos observar nestas figuras que reordenar a submissão de kernels tem um impacto significativo sobre o turnaround time médio do conjunto de kernels. As reduções em ANTT variam de $45 \%$ a $75 \%$. Podemos observar também que o conjunto de kernels com $N=256$ produziu ganhos mais pronunciados. Isso ocorre porque há mais itens para serem inseridos na mochila e o algoritmo tem mais possibilidades de enchê-la.

A Tabela 2 mostra os resultados de STP para as mesmas variações de $N$ e $N B$. 


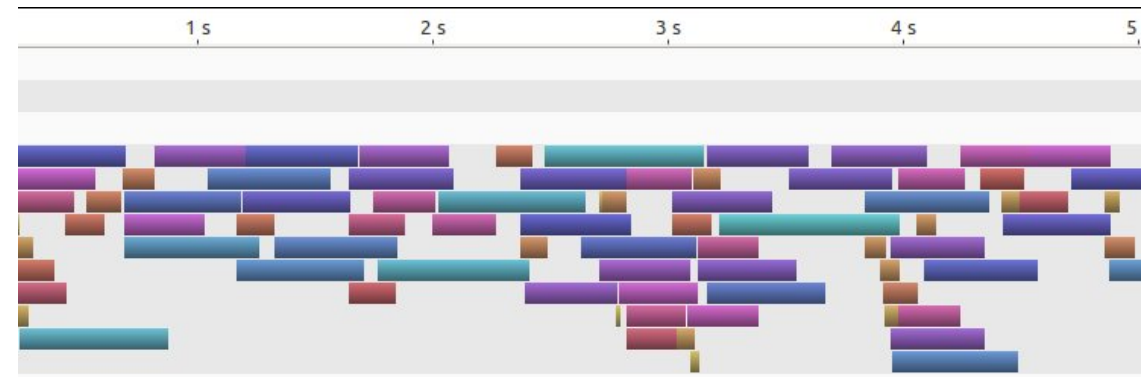

Figura 2. Profile de execução de 128 kernels com submissão padrão.

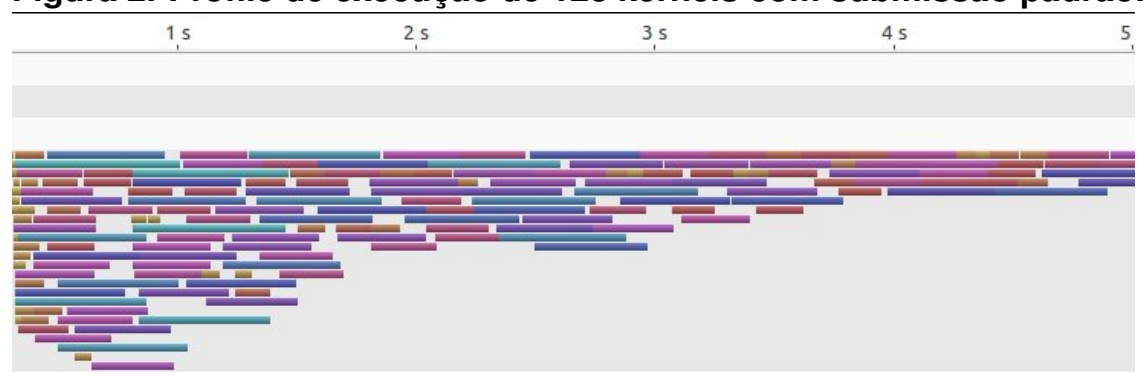

Figura 3. Profile de execução de 128 kernels com submissão reordenada.

Observamos nesta tabela que o throughput é consideravelmente maior quando nosso esquema de reordenação é utilizado. Nossa abordagem aumenta o STP de 1,6 a 3,5 vezes, o que significa que ele está melhorando a utilização da GPU. Ilustramos essa utilização nas Figuras 2 e 3 que mostram um corte dos resultados (entre de 0 a 5s) do CUDA Profiler para $N=128$ e $N B=32$. Podemos observar que a execução padrão permite um máximo de 10 núcleos executando simultaneamente, enquanto que a execução com reordenação permite até 24 kernels simultâneos e um consequente aumento do uso de recursos.

\begin{tabular}{|c|c|c|c|c|c|c|c|c|}
\hline \multirow{3}{*}{ NB } & \multicolumn{90}{|c|}{ STP } \\
\cline { 2 - 9 } & \multicolumn{2}{|c|}{32 Kernels } & \multicolumn{2}{|c|}{ 64 Kernels } & \multicolumn{2}{c|}{128 Kernels } & \multicolumn{2}{c|}{ 256 Kernels } \\
\cline { 2 - 9 } & Padrão & Reord. & Padrão & Reord. & Padrão & Reord. & Padrão & Reord. \\
\hline 32 & 11 & 18 & 14 & 25 & 16 & 39 & 21 & 54 \\
\hline 64 & 7.6 & 13 & 9.3 & 19 & 10 & 29 & 12 & 43 \\
\hline 128 & 5.6 & 10 & 8.0 & 14 & 8.8 & 23 & 9.6 & 28 \\
\hline 256 & 5.0 & 8.2 & 5.5 & 10 & 6.6 & 17 & 7.1 & 22 \\
\hline 512 & 4.2 & 6.6 & 5.0 & 8.9 & 5.4 & 12 & 6.5 & 16 \\
\hline
\end{tabular}

Tabela 2. Resultados de STP para diferentes valores de $N$ and $N B$.

\subsection{Overhead}

A reordenação é executada estaticamente antes que os kernels sejam submetidos à GPU. No entanto, uma vez que o cálculo da reordenação envolve a solução de um problema de otimização combinatória, avaliamos a overhead do algoritmo de reordenação. A Tabela 3 mostra a overhead de nossa abordagem de reordenação para $N \in\{32,64,128,256\} \mathrm{e}$ $N B \in\{32,64,128,256,512\}$. O overhead é calculado comparando o tempo de execução do algoritmo de reordenação com o ganho absoluto no turnaround time médio gerado por reordenar os kernels. O overhead mostra a porcentagem de nossos ganhos que foi gasta em computar a solução. 


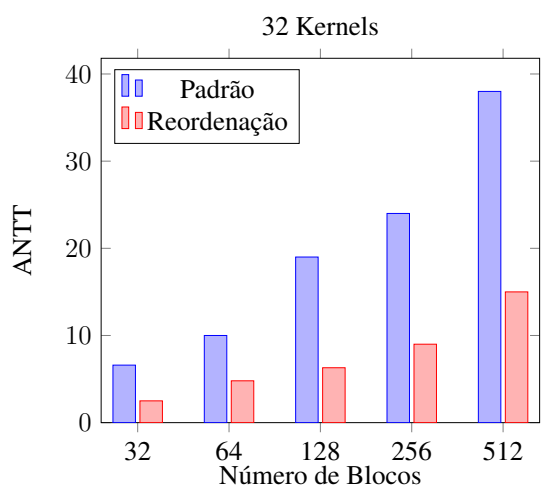

Figura 4. Resultados de ANTT para $\mathrm{N}=32$ na TITAN $\mathrm{X}$.

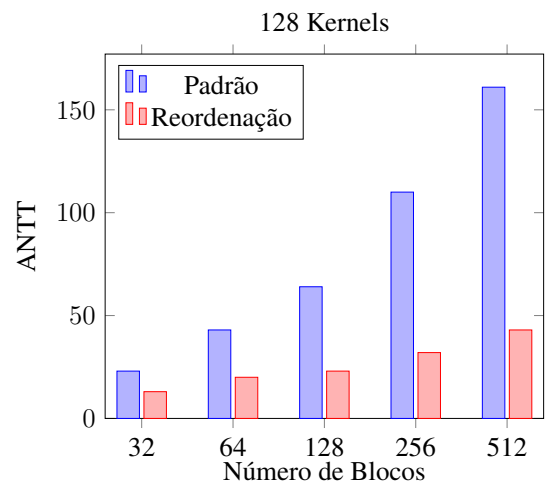

Figura 6. Resultados de ANTT para $\mathrm{N}=128$ na TITAN $\mathrm{X}$.

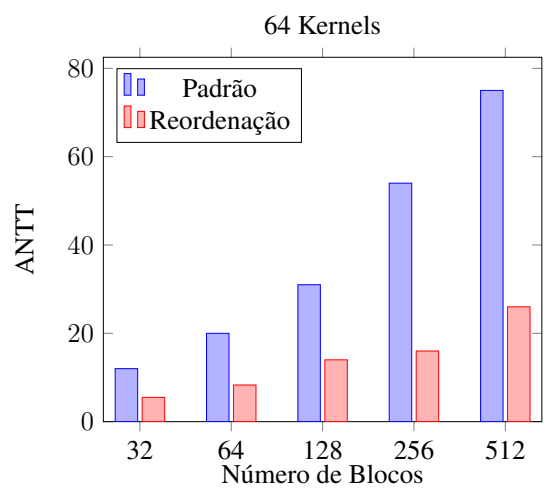

Figura 5. Resultados de ANTT para $\mathrm{N}=64$ na TITAN $\mathrm{X}$.

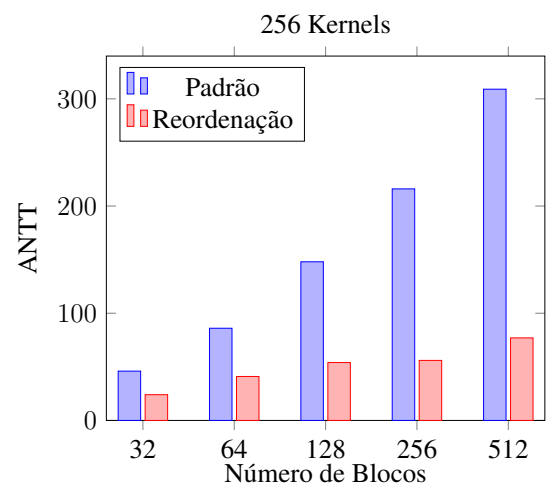

Figura 7. Resultados de ANTT para $\mathrm{N}=256$ na TITAN $\mathrm{X}$.

Podemos observar na Tabela 3 que os overheads são baixos. Gastamos de 0,1\% para $6,3 \%$ dos ganhos para calcular a reordenação. Também podemos observar que os maiores overheads foram encontrados com um pequeno número de kernels e blocos. Isso ocorre porque a pequena quantidade de computação leva a ganhos menores no tempo médio. Para maior número de kernels e blocos, o overhead de resolver os problemas da mochila torna-se insignificante.

\begin{tabular}{|c|c|c|c|c|}
\hline \multirow{2}{*}{ NB } & \multicolumn{4}{|c|}{ Overhead } \\
\cline { 2 - 5 } & 32 Kernels & 64 Kernels & 128 Kernels & 256 Kernels \\
\hline 32 & 6.3 & 2.1 & 2.1 & 2.5 \\
\hline 64 & 2.7 & 1.0 & 1.0 & 1.3 \\
\hline 128 & 0.5 & 0.5 & 0.5 & 0.8 \\
\hline 256 & 0.4 & 0.2 & 0.2 & 0.4 \\
\hline 512 & 0.2 & 0.2 & 0.2 & 0.2 \\
\hline
\end{tabular}

Tabela 3. Overhead da Reordenação.

\subsection{Portabilidade}

Nosso esquema de reordenação é uma solução de software que pode ser aplicada a diferentes arquiteturas da NVIDIA que permitam a existência de kernels concorrentes. Avaliamos também nossa abordagem usando uma GPU baseada na arquitetura Kepler. Apresentamos nas Tabelas 4 a 7 os resultados de ANTT e STP para a GPU K40. Nossa 
solução atinge reduções substanciais em ANTT e melhorias em STP quando comparada com a submissão padrão. Obtivemos de $31 \%$ a $73 \%$ de redução em ANTT, e STP foi melhorado de 1,1 a 3,2 vezes. Comparando com os resultados da TITAN-X, observamos ganhos semelhantes.

\begin{tabular}{|c|c|c|c|c|}
\hline \multirow{2}{*}{ NB } & \multicolumn{2}{|c|}{ ANTT } & \multicolumn{2}{c|}{ STP } \\
\cline { 2 - 5 } & Padrão & Reord & Padrão & Reord \\
\hline 32 & 17 & 8.5 & 6.2 & 8.7 \\
\hline 64 & 18 & 10 & 5.0 & 7.9 \\
\hline 128 & 28 & 17 & 4.5 & 6.9 \\
\hline 256 & 50 & 34 & 4.1 & 5.8 \\
\hline 512 & 53 & 35 & 4.1 & 4.5 \\
\hline
\end{tabular}

Tabela 4. Execução com 32 kernels.

\begin{tabular}{|c|c|c|c|c|}
\hline \multirow{2}{*}{ NB } & \multicolumn{2}{|c|}{ ANTT } & \multicolumn{2}{c|}{ STP } \\
\cline { 2 - 5 } & Padrão & Reord & Padrão & Reord \\
\hline 32 & 56 & 23 & 8.4 & 21 \\
\hline 64 & 91 & 32 & 6.9 & 18 \\
\hline 128 & 133 & 40 & 5.6 & 13 \\
\hline 256 & 166 & 59 & 5.9 & 11 \\
\hline 512 & 220 & 76 & 5.6 & 10 \\
\hline
\end{tabular}

Tabela 6. Execução com 128 kernels.

\begin{tabular}{|c|c|c|c|c|}
\hline \multirow{2}{*}{ NB } & \multicolumn{2}{|c|}{ ANTT } & \multicolumn{2}{c|}{ STP } \\
\cline { 2 - 5 } & Padrão & Reord & Padrão & Reord \\
\hline 32 & 28 & 12 & 7.1 & 15 \\
\hline 64 & 49 & 16 & 6.3 & 12 \\
\hline 128 & 68 & 22 & 4.7 & 9.4 \\
\hline 256 & 98 & 25 & 4.9 & 7.6 \\
\hline 512 & 116 & 35 & 5.0 & 7.5 \\
\hline
\end{tabular}

Tabela 5. Execução com 64 kernels.

\begin{tabular}{|c|c|c|c|c|}
\hline \multirow{2}{*}{ NB } & \multicolumn{2}{|c|}{ ANTT } & \multicolumn{2}{c|}{ STP } \\
\cline { 2 - 5 } & Padrão & Reord & Padrão & Reord \\
\hline 32 & 115 & 50 & 9.2 & 29 \\
\hline 64 & 183 & 64 & 7.4 & 22 \\
\hline 128 & 261 & 85 & 6.9 & 17 \\
\hline 256 & 355 & 108 & 6.1 & 13 \\
\hline 512 & 419 & 143 & 6.3 & 12 \\
\hline
\end{tabular}

Tabela 7. Execução com 128 kernels.

\section{Conclusões}

Como o hardware da GPU continua evoluindo, oferecendo uma quantidade cada vez maior de recursos, é muito provável que, no futuro, a GPU avance para um dispositivo multiprogramado. Neste cenário, o compartilhamento dos recursos da GPU é a chave para melhorar o throughput. Neste trabalho, propomos uma estratégia de reordenação dos kernels submetidos para executar na GPU. Nossa ideia é resolver uma série de problemas da mochila para encontrar os kernels que maximizam a utilização GPU. Toda vez que há recursos disponíveis, o algoritmo modela a quantidade de recursos como a capacidade da mochila e tenta encher a mochila com os kernels que levam ao máximo proveito desses recursos disponíveis, favorecendo os kernels com menor tempo de execução.

Apresentamos uma série de experimentos utilizando kernels sintéticos que requerem diferentes quantidades de recursos. Nossos resultados mostram que a reordenação proporciona ganhos significativos no tempo médio de turnaround e no throughput do sistema em comparação com a submissão de kernels padrão implementada em GPUs modernas. O overhead do algoritmo de reordenação é insignificante em comparação com os ganhos no tempo médio de execução. Também mostramos que a nossa abordagem proporciona ganhos semelhantes nas GPUs Kepler e Maxwell. Futuramente, pretendemos incorporar o nosso esquema de reordenação no serviço de multi-processamento NVIDIA (MPS). O uso de benchmarks da litaretura é um passo futuro importante para validar o modelo em diferentes cenários.

\section{Agradecimentos}

Este trabalho foi parcialmente financiado com recursos da NVIDIA, CNPq, CAPES e FAPERJ. 


\section{Referências}

Adriaens, J. T., Compton, K., Kim, N. S., and Schulte, M. J. (2012). The case for GPGPU spatial multitasking. In IEEE 18th International Symposium on High Performance Computer Architecture (HPCA), pages 1-12. IEEE.

Choi, H. J., Son, D. O., Kang, S. G., Kim, J. M., Lee, H.-H., and Kim, C. H. (2013). An efficient scheduling scheme using estimated execution time for heterogeneous computing systems. The Journal of Supercomputing, 65(2):886-902.

Eyerman, S. and Eeckhout, L. (2008). System-level performance metrics for multiprogram workloads. IEEE Micro, 28(3):42-53.

Gregg, C., Dorn, J., Hazelwood, K., and Skadron, K. (2012). Fine-grained resource sharing for concurrent GPGPU kernels. In Presented as part of the 4th USENIX Workshop on Hot Topics in Parallelism.

Li, T., Narayana, V. K., and El-Ghazawi, T. (2015). A power-aware symbiotic scheduling algorithm for concurrent GPU kernels. In The 21st IEEE International Conference on Parallel and Distributed Systems (ICPADS).

Liang, Y., Huynh, P., Rupnow, K., Goh, R., and Chen, D. (2015). Efficient GPU spatialtemporal multitasking. IEEE Trans. on Parallel and Distributed Systems, 26:748-760.

Lopez-Novoa, U., Mendiburu, A., and Miguel-Alonso, J. (2015). A survey of performance modeling and simulation techniques for accelerator-based computing. IEEE Transactions on Parallel and Distributed Systems, 26(1):272-281.

Martello, S. and Toth, P. (1990). Knapsack problems: algorithms and computer implementations. John Wiley \& Sons, Inc.

NVIDIA (2016). Cuda Profiler. http://docs.nvidia.com/cuda/profiler-users-guide.

Pai, S., Thazhuthaveetil, M. J., and Govindarajan, R. (2013). Improving GPGPU concurrency with elastic kernels. In ACM SIGPLAN Notices, volume 48, pages 407-418.

Peters, H., Koper, M., and Luttenberger, N. (2010). Efficiently using a CUDA-enabled GPU as shared resource. In IEEE 10th International Conference on Computer and Information Technology (CIT), pages 1122-1127. IEEE.

Ravi, V. T., Becchi, M., Agrawal, G., and Chakradhar, S. (2011). Supporting GPU sharing in cloud environments with a transparent runtime consolidation framework. In Proceedings of the 20th international symposium on High performance distributed computing, pages 217-228. ACM.

Wang, L., Huang, M., and El-Ghazawi, T. (2011). Exploiting concurrent kernel execution on graphic processing units. In International Conference on High Performance Computing and Simulation (HPCS), pages 24-32. IEEE.

Wende, F., Cordes, F., and Steinke, T. (2012). On improving the performance of multithreaded CUDA applications with concurrent kernel execution by kernel reordering. In Symp. on Application Accelerators in High Performance Computing (SAAHPC),74-83.

Zhong, J. and He, B. (2014). Kernelet: High-throughput GPU kernel executions with dynamic slicing and scheduling. IEEE Transactions on Parallel and Distributed Systems, 25(6):1522-1532. 